\title{
McDonald log-logistic distribution with an application to breast cancer data
}

\author{
M. H. Tahir \\ Department of Statistics \\ The Islamia University of Bahawalpur, Bahawalpur-63100, Pakistan \\ mtahir.stat@gmail.com \\ Muhammad Mansoor \\ Department of Statistics \\ Punjab College, Bahawalpur-63100, Pakistan \\ mansoor.abbasi143@gmail.com \\ Muhammad Zubair \\ Department of Statisticss \\ Government Degree College Kahrorpacca, Lodhran-59320, Pakistan \\ zubair.stat@yahoo.com \\ G. G. Hamedani \\ Department of Mathematics, Statistics and Computer Science \\ Marquette University, Milwaukee, USA \\ g.hamedani@mu.edu
}

Received 19 October 2013

Accepted 21 February 2014

\begin{abstract}
We introduce a five-parameter continuous model, called the McDonald log-logistic distribution, to extend the two-parameter log-logistic distribution. Some structural properties of this new distribution such as reliability measures and entropies are obtained. The model parameters are estimated by the method of maximum likelihood using L-BFGS-B algorithm. A useful characterization of the distribution is proposed which does not require explicit closed form of the cumulative distribution function and also connects the probability density function with a solution of a first order differential equation. An application of the new model to real data set shows that it can give consistently better fit than other important lifetime models.
\end{abstract}

Keywords: Log-logistic distribution; hazard function; reliability function, Rényi entropy.

2000 Mathematics Subject Classification: 60E05; 62E10; 62N05

\section{Introduction}

The Log-Logistic ("LL" for short) distribution is a very popular logistic distribution which was initially developed to model population growth by Verhulst (1838). In income inequality literature, LL distribution is well-known as Fisk distribution due to Fisk (1961), and has also been widely used in many areas such as reliability, survival analysis, actuarial science, economics, engineering and hydrology. In some cases, LL distribution is proved to be a good alterative to the log-normal distribution since it characterize increasing hazard rate function. Further, its use is well appreciated in case of censored data usually common in reliability and life-testing experiments. 
The paper involves the use of the following well-known functions and series expansion expressions, including the beta function of type I is defined by

$$
B(p, q)=\int_{0}^{1} t^{p-1}(1-t)^{q-1} d t,
$$

the incomplete beta function is defined by

$$
B_{x}(p, q)=\int_{0}^{x} t^{p-1}(1-t)^{q-1} d t,
$$

the incomplete beta function ratio of type I is defined by

$$
I_{x}(p, q)=\frac{1}{B(p, q)} \int_{0}^{x} t^{p-1}(1-t)^{q-1} d t=\frac{B_{x}(p, q)}{B(p, q)},
$$

the complementary incomplete beta function of type I is defined by

$$
B(p, q ; x)=\int_{x}^{1} t^{q-1}(1-t)^{q-1} d t,
$$

the beta function of type II is defined by

$$
B(p, q)=\int_{0}^{\infty} t^{p-1}(1+t)^{-(p+q)} d t
$$

the gamma function is defined by

$$
\Gamma(p)=\int_{0}^{\infty} t^{p-1} e^{-t} d t
$$

the incomplete gamma function is defined by

$$
\gamma(p, x)=\int_{0}^{x} t^{p-1} e^{-t} d t
$$

the binomial series expansion is defined by

$$
(1-Z)^{m}=\sum_{j=0}^{\infty}(-1)^{j}\left(\begin{array}{c}
m \\
j
\end{array}\right) Z^{j}=\sum_{j=0}^{\infty}(-1)^{j} \frac{\Gamma(m+1)}{\Gamma(m-j+1)} \frac{z^{j}}{j !} .
$$

The probability density function (pdf) and the cumulative distribution function (cdf) of LL distribution with shape parameter $\alpha$ and scale parameter $\beta$ are given by

$$
g(x)=\left(\frac{\alpha}{\beta}\right)\left(\frac{x}{\beta}\right)^{\alpha-1}\left[1+\left(\frac{x}{\beta}\right)^{\alpha}\right]^{-2}, \quad x>0, \quad \alpha, \beta>0
$$

and

$$
G(x)=1-\left[1+\left(\frac{x}{\beta}\right)^{\alpha}\right]^{-1}=\left(\frac{x}{\beta}\right)^{\alpha}\left[1+\left(\frac{x}{\beta}\right)^{\alpha}\right]^{-1}, \quad x>0, \quad \alpha, \beta>0 .
$$

There is an increasing trend in the extension (or generalization) of the baseline distribution by adding shape parameter(s) to the baseline distribution. In literature, three popular extended models of LL exist Viz. Beta Log-Logistic (BLL), Kumaraswamy Log-Logistic (KwLL) and ZografosBalakrishnan (2009) Log-Logistic (ZBLL). 
We first consider the BLL distribution, which is generated from beta-generated (Beta-G) class of distributions introduced by Eugene et al. (2002), and further discussed by Jones (2004).

For any arbitrary baseline pdf $g(x)$ and cdf $G(x)$, the pdf $f(x)$ and cdf $F(x)$ of Beta-G class of distributions are defined by

$$
f(x)=\frac{1}{B(a, b)} g(x)\{G(x)\}^{a-1}\{1-G(x)\}^{b-1}
$$

and

$$
F(x)=I_{G(x)}(a, b),
$$

where $a>0, b>0$ and are both shape parameters.

Lemonte (2012) introduced the pdf and cdf of BLL distribution, which are given by

$$
f(x)=\frac{1}{B(a, b)}\left(\frac{\alpha}{\beta}\right)\left(\frac{x}{\beta}\right)^{a \alpha-1}\left[1+\left(\frac{x}{\beta}\right)^{\alpha}\right]^{-(a+b)}, \quad x>0
$$

and

$$
F(x)=I_{\left[1-\left[1+(x / \beta)^{\alpha}\right]^{-1}\right]}(a, b) \quad x>0,
$$

where $a, b, \alpha, \beta>0$, and $a, b$ and $\alpha$ are shape parameters while $\beta$ is scale parameter.

Now, we consider the second extended model, the KwLL distribution, which is based on the Kumaraswamy generalized (Kw-G) class of distributions.

For a baseline random variable having pdf $g(x)$ and cdf $G(x)$, Cordeiro and de Castro (2011) defined the 2-parameter Kw-G pdf and cdf are defined by

$$
f(x)=a b g(x)[G(x)]^{a-1}\left[1-G(x)^{a}\right]^{b-1},
$$

and

$$
F(x)=1-\left[1-G(x)^{a}\right]^{b},
$$

where $g(x)=d G(x) / d x$ and $a>0$ and $b>0$ are two additional shape parameters whose role are to govern skewness and tail weights.

de Santana et al. (2012) introduced the pdf and cdf of KwLL distribution, which are given by

$$
f(x)=a b\left(\frac{\alpha}{\beta}\right)\left(\frac{x}{\beta}\right)^{a \alpha-1}\left[1+\left(\frac{x}{\beta}\right)^{\alpha}\right]^{-(a+1)}\left[1-\left\{1-\frac{1}{1+\left(\frac{x}{\beta}\right)^{\alpha}}\right\}^{a}\right]^{b-1}, \quad x>0
$$

and

$$
F(x)=1-\left[1-\left\{1-\frac{1}{1+\left(\frac{x}{\beta}\right)^{\alpha}}\right\}^{a}\right]^{b}, \quad x>0
$$

where $a, b, \alpha, \beta>0$ and $a, b$ and $\alpha$ are shape parameters while $\beta$ is scale parameter. 
Now, we consider the third extended model, the ZBLL distribution, which is based on the Zografos and Balakrishnan (2009)' gamma-generated ('ZB-GG" for short) class of distributions.

For a baseline random variable having pdf $g(x)$ and cdf $G(x)$, Zografos and Balakrishnan (2009) defined the ZB-GG pdf $f(x)$ and $\operatorname{cdf} F(x)$ as

$$
f(x)=\frac{1}{\Gamma(a)}\{-\log [1-G(x)]\}^{a-1} g(x)
$$

and

$$
F(x)=\frac{1}{\Gamma(a)} \int_{0}^{-\log [1-G(x)]} t^{a-1} e^{-t} d t
$$

where $a>0$ and $g(x)=d G(x) / d x$.

Recently, Ramos et al. (2013) introduced the pdf and cdf of ZBLL distribution, which are given by

$$
f(x)=\frac{\alpha}{\beta^{\alpha} \Gamma(a)} x^{\alpha-1}\left[1+\left(\frac{x}{\beta}\right)^{\alpha}\right]^{-2}\left[\log \left\{1+\left(\frac{x}{\beta}\right)^{\alpha}\right\}\right]^{a-1}, \quad x>0
$$

and

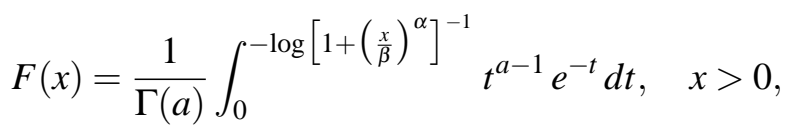

where $a, \alpha, \beta>0$.

The article is outlined as follows. In Section 2, we define the McLL distribution. Section 3 provides some new structural properties such as quantile function and mode. In Section 4, the expressions for mean residual life and mean time are obtained. In Section 5, the Rényi and $q$ entropies are derived. The maximum likelihood estimation of parameters is discussed in Section 6. A useful characterization of the distribution is introduced in Section 7. An empirical application is presented and discussed in Section 8. Finally, Section 9 offers some concluding remarks.

\section{McDonald Log-Logistic (McLL) distribution}

The generalized beta of first kind (GB1) or McDonald distribution was introduced by McDonald in 1984. The pdf and cdf of McDonald ("Mc" for short) distribution are given by

$$
f(x)=\frac{c}{B\left(a c^{-1}, b\right)} x^{a-1}\left(1-x^{c}\right)^{b-1}, \quad 0<x<1
$$

and

$$
F(x)=I_{x^{c}}\left(a c^{-1}, b\right),
$$

where $a>0, b>0$ and $c>0$ are shape parameters. 
For any arbitrary baseline pdf $g(x)$ and $\operatorname{cdf} G(x)$, Alexander et al. (2012) defined the pdf and cdf of McDonald-generalized (Mc-G) class of distribtions as

$$
f(x)=\frac{c}{B\left(a c^{-1}, b\right)} g(x)\{G(x)\}^{a-1}\left\{1-G(x)^{c}\right\}^{b-1}
$$

and

$$
F(x)=I_{G(x)^{c}}\left(a c^{-1}, b\right) .
$$

Now, inserting (1.9) and (1.10) in (2.3) and (2.4) to obtain the pdf and cdf of McLL distribution, respectively, as

$$
f(x)=\frac{c}{B\left(a c^{-1}, b\right)}\left(\frac{\alpha}{\beta}\right)\left(\frac{x}{\beta}\right)^{a \alpha-1}\left[1+\left(\frac{x}{\beta}\right)^{\alpha}\right]^{-(a+1)}\left[1-\left\{1-\left[1+\left(\frac{x}{\beta}\right)^{\alpha}\right]^{-1}\right\}^{c}\right]^{b-1}, \quad x>0
$$

and

$$
F(x)=I_{w}\left(a c^{-1}, b\right), \quad x>0,
$$

with $w=\left\{1-\left[1+\left(\frac{x}{\beta}\right)^{\alpha}\right]^{-1}\right\}^{c}$ and $a, b, c, \alpha, \beta>0$, where $a, b, c$ and $\alpha$ are shape parameters while $\beta$ is scale parameter.

We would like to point out the McLL is a special case of Mc-G when $G(x)=$ $\left\{1-\left[1+\left(\frac{x}{\beta}\right)^{\alpha}\right]^{-1}\right\}$. In the present work we discuss some further properties of McLL which have not appeared in Alexander et al. (2012) or anywhere else to our knowledge. Furthermore, we provide, among other new properties, a practical application of McLL in life-model case.

Plots of McLL density function for some parametric values are displayed in Figure 1.

For a lifetime random variable $t$, the survival function, $S(t)$, hazard rate function, $h(t)$, reversed hazard rate function, $r(t)$, and the cumulative hazard rate function, $H(t)$, of McLL distribution are given by

$$
\begin{gathered}
S(t)=1-F(t)=1-I_{w}\left(a c^{-1}, b\right), \\
h(t)=\frac{f(t)}{S(t)}=\frac{c\left(\frac{\alpha}{\beta}\right)\left(\frac{t}{\beta}\right)^{a \alpha-1}\left[1-\left\{1-\left[1+\left(\frac{t}{\beta}\right)^{\alpha}\right]^{-1}\right\}^{c}\right]^{b-1}}{B\left(a c^{-1}, b\right)\left[1+\left(\frac{t}{\beta}\right)^{\alpha}\right]^{(a+1)}\left[1-I_{w}\left(a c^{-1}, b\right)\right]} \\
r(t)=\frac{f(t)}{F(t)}=\frac{c\left(\frac{\alpha}{\beta}\right)\left(\frac{t}{\beta}\right)^{a \alpha-1}\left[1-\left\{1-\left[1+\left(\frac{t}{\beta}\right)^{\alpha}\right]^{-1}\right\}^{c}\right]^{b-1}}{B\left(a c^{-1}, b\right)\left[1+\left(\frac{t}{\beta}\right)^{\alpha}\right]^{(a+1)}\left[I_{w}\left(a c^{-1}, b\right)\right]}
\end{gathered}
$$

and

$$
H(t)=\int_{0}^{t} h(t) d t=-\ln S(t)=-\ln \left[1-I_{w}\left(a c^{-1}, b\right)\right] .
$$

Plots of hazard and reversed hazard rate functions for McLL density function for some parametric values are given in Figure 2. 
M.H. Tahir, M. Mansoor, M. Zubair and G.G. Hamedani

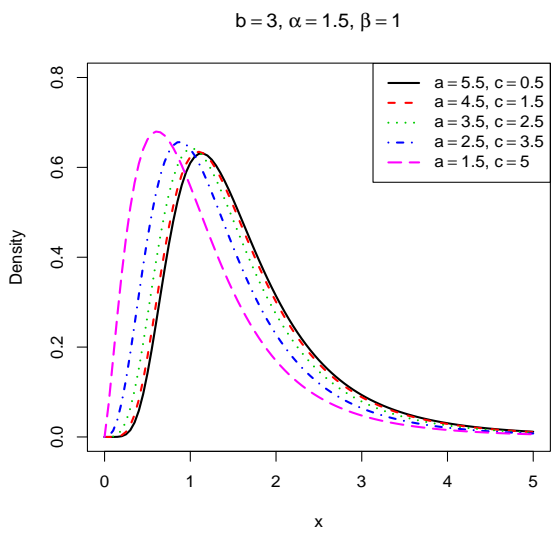

(a)

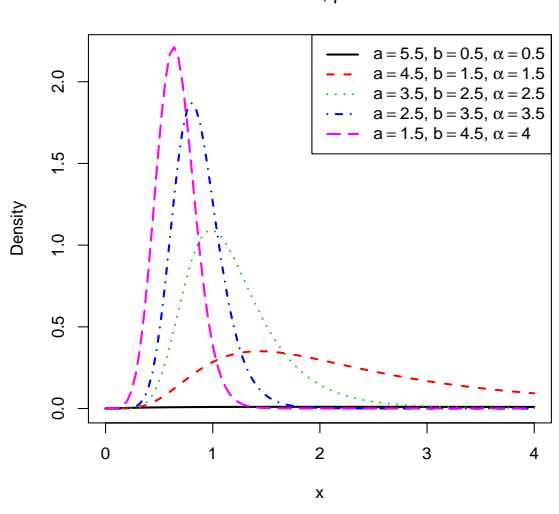

(c)

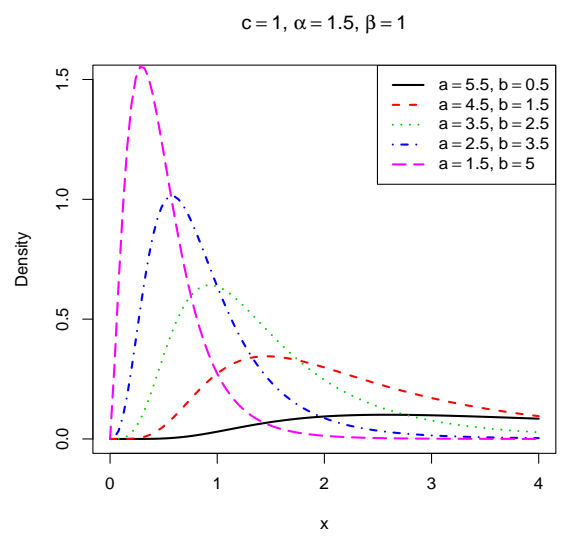

(b)

$\mathrm{C}=1, \alpha=1.5, \beta=1$

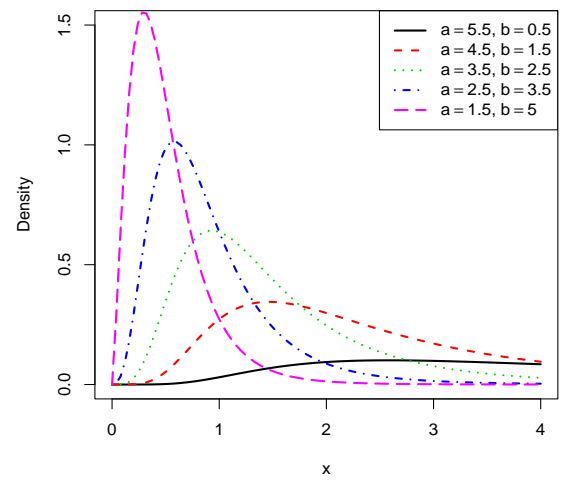

(d)

Fig. 1. Plots of McLL density for some parameter values

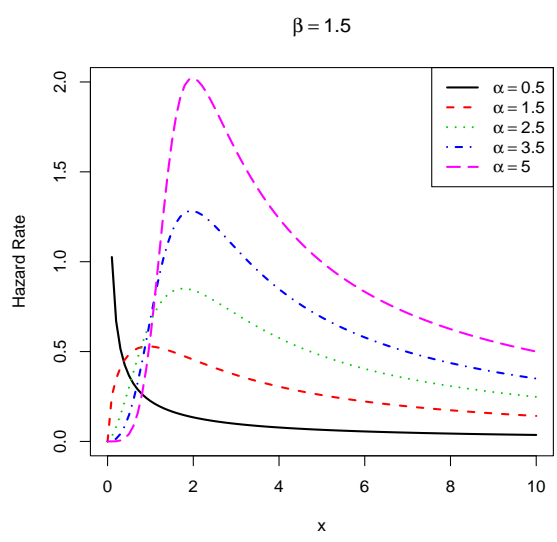

(e)

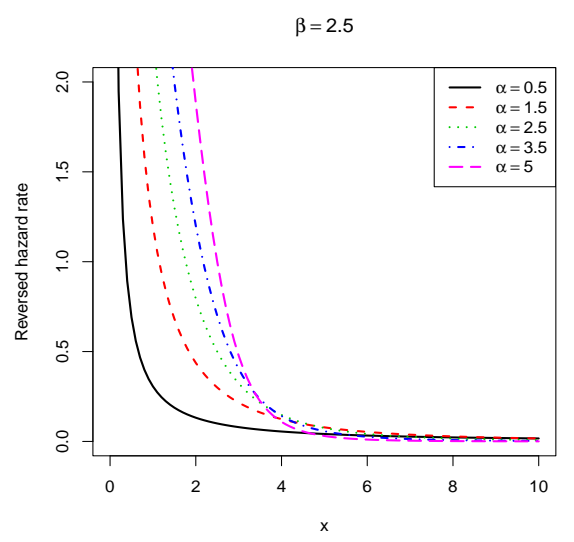

(f)

Fig. 2. Plots of (e) hazard and (f) reversed hazard rate for McLL model 


\subsection{Special sub-models}

The McLL distribution is very flexible and has the following distributions as special sub-models.

\subsubsection{Beta Log-Logistic distribution}

If $c=1$, the McLL distribution reduces to the BLL distribution with parameters $a, b, \alpha$ and $\beta$.

$$
f(x)=\frac{1}{B(a, b)}\left(\frac{\alpha}{\beta}\right)\left(\frac{x}{\beta}\right)^{a \alpha-1}\left[1+\left(\frac{x}{\beta}\right)^{\alpha}\right]^{-(a+b)}, \quad x>0,
$$

\subsubsection{Kumaraswamy Log-Logistic distribution}

If $c=a$, the McLL distribution reduces to the KwLL distribution with parameters $a, b, \alpha$ and $\beta$.

$$
f(x)=a b\left(\frac{\alpha}{\beta}\right)\left(\frac{x}{\beta}\right)^{a \alpha-1}\left[1+\left(\frac{x}{\beta}\right)^{\alpha}\right]^{-(a+1)}\left[1-\left\{1-\left[1+\left(\frac{x}{\beta}\right)^{\alpha}\right]^{-1}\right\}^{a}\right]^{b-1}, \quad x>0 .
$$

\subsubsection{Exponentiated Log-Logistic (Lehmann type I) or Dagum distribution}

If $b=c=1$, the McLL distribution reduces to the Exponentiated LL (Lehmann type I) distribution with parameters $a, \alpha$ and $\beta$.

$$
f(x)=a\left(\frac{\alpha}{\beta}\right)\left(\frac{x}{\beta}\right)^{a \alpha-1}\left[1+\left(\frac{x}{\beta}\right)^{\alpha}\right]^{-(a+1)}, \quad x>0 .
$$

\subsubsection{Exponentiated Log-Logistic Lehmann type II) or Singh-Maddala distribution}

If $a=c=1$, the McLL distribution reduces to the exponentiated LL (or Lehmann type II) distribution with parameters $b, \alpha$ and $\beta$.

$$
f(x)=b\left(\frac{\alpha}{\beta}\right)\left(\frac{x}{\beta}\right)^{\alpha-1}\left[1+\left(\frac{x}{\beta}\right)^{\alpha}\right]^{-(b+1)}, \quad x>0 .
$$

\subsubsection{Log-logistic distribution}

If $a=b=c=1$, the McLL distribution reduces to the LL distribution with parameters $\alpha$ and $\beta$.

$$
f(x)=\left(\frac{\alpha}{\beta}\right)\left(\frac{x}{\beta}\right)^{\alpha-1}\left[1+\left(\frac{x}{\beta}\right)^{\alpha}\right]^{-2}, \quad x>0 .
$$

\subsubsection{Standard log-logistic distribution}

If $a=b=c=\alpha=\beta=1$, the McLL distribution reduces to the standard LL distribution.

$$
f(x)=[1+x]^{-2}, \quad x>0 .
$$


M.H. Tahir, M. Mansoor, M. Zubair and G.G. Hamedani

2.2. Expansion of the McLL density

Re-calling the general form of McLL density, we have

$$
f(x)=\frac{c}{B\left(a c^{-1}, b\right)} g(x)[G(x)]^{a-1} \underbrace{\left[1-G(x)^{c}\right]^{b-1}}_{A}
$$

Using the binomial expansion (1.8) to result $\mathrm{A}$ in (2.11) when $b$ is real non-integer and $|Z|<1$, the (2.11) now can be expressed as

$$
f(x)=\frac{c}{B\left(a c^{-1}, b\right)} g(x)[G(x)]^{a-1} \sum_{i=0}^{\infty}(-1)^{i}\left(\begin{array}{c}
b-1 \\
i
\end{array}\right)[G(x)]^{i c} .
$$

Simplifying further to (2.12), we have

$$
f(x)=g(x) \sum_{i=0}^{\infty} \underbrace{\frac{c}{B\left(a c^{-1}, b\right)}(-1)^{i}\left(\begin{array}{c}
b-1 \\
i
\end{array}\right)}_{W_{i}}[G(x)]^{i c+a-1},
$$

where $a$ is real non-integer.

Using binomial expansion (1.8) to the term $[G(x)]^{i c+a-1}$ in (2.13), we have

$$
\begin{aligned}
{[G(x)]^{i c+a-1} } & =[1-[1-G(x)]]^{i c+a-1} \\
& =\underbrace{\sum_{k=j}^{\infty}(-1)^{j+k}\left(\begin{array}{c}
i c+a-1 \\
j
\end{array}\right)\left(\begin{array}{l}
j \\
k
\end{array}\right)}_{S_{k}}[G(x)]^{k} \\
& =S_{k}[G(x)]^{k}
\end{aligned}
$$

Now, (2.12) becomes

$$
f(x)=g(x) \sum_{k=0}^{\infty} \underbrace{W_{i} S_{k}}_{P_{k}}[G(x)]^{k} .
$$

Finally, we can write the McLL density as

$$
f(x)=\underbrace{g(x)}_{\text {density of } \mathrm{LL}} \underbrace{\sum_{k=0}^{\infty} P_{k}}_{\text {sum of weights }} \underbrace{[G(x)]^{k}}_{(\text {cdf of LL) }} .
$$

\section{Basic Properties}

In this section we deal with the basic statistical properties of McLL distribution. Some basic properties such as rth moment, mean, variance, generating function and characteristic function have been 
given by Alexander et al. (2012), some of which are as under:

$$
\begin{gathered}
\mu_{r}^{\prime}=\sum_{k=0}^{\infty} P_{k} \beta^{r} B\left(1+\frac{r}{\alpha}+k, 1-\frac{r}{\alpha}\right) \quad r=1,2, \ldots, \\
\mu=E(x)=\sum_{k=0}^{\infty} P_{k} \beta B\left(1+\frac{1}{\alpha}+k, 1-\frac{1}{\alpha}\right)
\end{gathered}
$$

and

$$
\text { Variance }=\sum_{k=0}^{\infty} P_{k} \beta^{2}\left[B\left(1+\frac{2}{\alpha}+k, 1-\frac{2}{\alpha}\right)-B^{2}\left(1+\frac{1}{\alpha}+k, 1-\frac{1}{\alpha}\right)\right]
$$

So, the other important basic properties such as quantile function and mode of the McLL distribution are mathematically obtained below.

\subsection{Quantile function and mode}

Let $Q_{\alpha, \beta}(p)$ be the quantile function of the LL distribution with parameters $\alpha$ and $\beta$, the quantile function of the $\operatorname{McLL}(a, b, c, \alpha, \beta)$ distribution, say, $x=Q(p)$ can easily be obtained as

$$
x=Q(p)=\frac{Q_{\alpha, \beta}(p)\left[Q_{\left(a c^{-1}, b\right)}(p)\right]^{\frac{1}{c}}}{1-Q_{\alpha, \beta}(p)\left[Q_{\left(a c^{-1}, b\right)}(p)\right]^{\frac{1}{c}}}, \quad o<p<1 .
$$

The Bowley skewness measure and Moors kurtosis (based on octiles) of the McLL distribution can be calculated using the formulae given below:

$$
S k=\frac{Q(3 / 4)+Q(1 / 4)-2 Q(2 / 4)}{Q(3 / 4)-Q(1 / 4)}
$$

and

$$
M=\frac{Q(3 / 8)+Q(1 / 8)+Q(7 / 8)-Q(5 / 8)}{Q(6 / 8)-Q(2 / 8)} .
$$

where $Q($.$) denotes the quantile function.$

The first derivative of $\log f(x)$ for the McLL distribution is

$$
\begin{aligned}
\frac{d \log f(x)}{d x} & =\frac{a \alpha-1}{x}-(a+1)\left[\frac{\left(\frac{\alpha}{\beta}\right)\left(\frac{x}{\beta}\right)^{\alpha-1}}{1+\left(\frac{x}{\beta}\right)^{\alpha}}\right] \\
& -c(b-1)\left[\frac{\left(\frac{\alpha}{\beta}\right)\left(\frac{x}{\beta}\right)^{\alpha-1}\left\{1-\left[1+\left(\frac{x}{\beta}\right)^{\alpha}\right]^{-1}\right\}^{c-1}\left[1+\left(\frac{x}{\beta}\right)^{\alpha}\right]^{-2}}{1-\left\{1-\left[1+\left(\frac{x}{\beta}\right)^{\alpha}\right]^{-1}\right\}^{c}}\right] .
\end{aligned}
$$


So, the modes of the McLL distribution are the roots of the following equation

$$
\begin{aligned}
& \frac{a \alpha-1}{x}-(a+1)\left[\frac{\left(\frac{\alpha}{\beta}\right)\left(\frac{x}{\beta}\right)^{\alpha-1}}{1+\left(\frac{x}{\beta}\right)^{\alpha}}\right] \\
& =c(b-1)\left[\frac{\left(\frac{\alpha}{\beta}\right)\left(\frac{x}{\beta}\right)^{\alpha-1}\left\{1-\left[1+\left(\frac{x}{\beta}\right)^{\alpha}\right]^{-1}\right\}^{c-1}\left[1+\left(\frac{x}{\beta}\right)^{\alpha}\right]^{-2}}{1-\left\{1-\left[1+\left(\frac{x}{\beta}\right)^{\alpha}\right]^{-1}\right\}^{c}}\right] .
\end{aligned}
$$

There may be more than one root to the above equation. If $x=x_{0}$ is a root of the above equation, then it corresponds to a local maximum or local minimum or a point of inflexion depending on whether $\frac{d^{2} \log f(x)}{(d x)^{2}}<$ or $>$ or $=0$, respectively.

If $a=b=c=1$ in the above, we get the mode of the LL distribution.

\section{Mean Residual life and mean waiting time}

In this section, the reliability properties mean residual life function (MRL) and mean waiting time (MWT) are mathematically obtained for the McLL distribution.

The MRL at a given time $t$ measures the expected remaining lifetime of an individual of age $t$. It is denoted by $m(t)$. The MRL or life expectancy is defined as

$$
m(t)=\frac{1}{S(t)}\left[E(t)-\int_{0}^{t} t f(t) d t\right]-t .
$$

Solving the integral in (4.1), we obtain

$$
\int_{0}^{t} t f(t) d t=\beta \sum_{k=0}^{\infty} P_{k} B_{w}\left(1+\frac{1}{\alpha}+k, 1-\frac{1}{\alpha}\right)
$$

Inserting (4.2), (3.2) and (2.7) in (4.1), the MRL of the McLL distribution will be

$$
m(t)=\left[\frac{\beta \sum_{k=0}^{\infty} P_{k}\left\{B\left(1+\frac{1}{\alpha}+k, 1-\frac{1}{\alpha}\right)-B_{w}\left(1+\frac{1}{\alpha}+k, 1-\frac{1}{\alpha}\right)\right\}}{1-I_{w}\left(a c^{-1}, b\right)}\right]-t .
$$

The MWT of an item failed in a interval $[0, t]$ is defined as

$$
\bar{\mu}(t, \theta)=t-\left\{\frac{1}{F(t)} \int_{0}^{t} t f(t) d t\right\} .
$$

Using (4.2) and (2.6) in (4.4), the MWT of the McLL distribution obtained is

$$
\bar{\mu}(t, \theta)=t-\left[\frac{\sum_{k=0}^{\infty} P_{k} \beta B_{w}\left(1+\frac{1}{\alpha}+k, 1-\frac{1}{\alpha}\right)}{I_{w}\left(a c^{-1}, b\right)}\right] .
$$

\section{Entropies}

Entropy has wide application in science, engineering and probability theory, and has been used in various situations as a measure of variation of the uncertainty. Simply, an entropy of a random variable $X$ is a measure of uncertain amount of information in a distribution. Numerous measures of entropy have been studied and compared in the literature. Here, we derive explicit expressions for two important entropies for McLL distribution Viz. Rényi entropy and $q$-entropy. 


\subsection{Rényi entropy}

We first consider the Rényi entropy which plays a similar role as the kurtosis measure in comparing the shapes of various densities, and also measuring heaviness of tails. Rényi introduced an entropy in 1961 , which is defined by

$$
I_{R}(\gamma)=\frac{1}{1-\gamma} \log \left\{\int f^{\gamma}(x) d x\right\}
$$

where $\gamma>0, \gamma \neq 1$ and $\gamma$ is a real non-integer.

Now taking $\gamma$ th power of McLL density given in (2.5) as

$$
f^{\gamma}(x)=\left\{\frac{c}{B\left(a c^{-1}, b\right)}\left(\frac{\alpha}{\beta}\right)\left(\frac{x}{\beta}\right)^{a \alpha-1}\left[1+\left(\frac{x}{\beta}\right)^{\alpha}\right]^{-(a+1)}\left[1-\left\{1-\left[1+\left(\frac{x}{\beta}\right)^{\alpha}\right]^{-1}\right\}^{c}\right]^{b-1}\right\}^{\gamma} .
$$

Integrating (5.2), and then using (1.8), we get

$$
\int_{0}^{\infty} f^{\gamma}(x) d x=\sum_{j, k=0}^{\infty} V_{j} V_{k}\left(\frac{\alpha}{\beta}\right)^{\gamma}\left(\frac{x}{\beta}\right)^{\gamma(a \alpha-1)}\left[1+\left(\frac{x}{\beta}\right)^{\alpha}\right]^{-\gamma(a+1)-k} d x .
$$

Using transformation $\kappa=(x / \beta)^{\alpha}$ and then using (1.1), the (5.3) reduces to

$$
\int_{0}^{\infty} f^{\gamma}(x)=\sum_{j, k=0}^{\infty} V_{j} V_{k}\left(\frac{\alpha}{\beta}\right)^{\gamma-1} B\left(\frac{\gamma(\alpha-1)+1}{\alpha}, \gamma(a+1)+k-\frac{\gamma(\alpha-1)+1}{\alpha}\right)
$$

where $V_{j}=\left[\frac{c}{B\left(a c^{-1}, b\right)}\right]^{\gamma}(-1)^{j}\left(\begin{array}{c}\gamma(b-1) \\ j\end{array}\right)$ and $V_{k}=(-1)^{k}\left(\begin{array}{c}c_{j} \\ k\end{array}\right)$.

Now, inserting (5.4) in (5.1), the Rényi entropy expression for McLL distribution obtained is

$$
I_{R}(\gamma)=\frac{1}{1-\gamma} \log \left\{\sum_{j, k=0}^{\infty} V_{j} V_{k}\left(\frac{\alpha}{\beta}\right)^{\gamma-1} B\left(\frac{\gamma(\alpha-1)+1}{\alpha}, \gamma(a+1)+k-\frac{\gamma(\alpha-1)+1}{\alpha}\right)\right\} .
$$

\section{2. q-entropy}

The $q$ - (or $\tilde{\beta}$-entropy) was originally introduced by Havrda and Charvat (1967) and later applied to physical problems by Tsallis (1988). Tsallis exploited its non-extensive features and placed it in a physical setting (hence it is also known as Tsallis entropy). Moreover, $q$-entropy is a oneparameter generalization of the Shannon entropy which can lead to models or statistical results that are different from those obtained by using the Shannon entropy. It is to be noted here that the $q$-entropy is a monotonic function of the Rényi entropy (Ullah, 1996).

For a continuous random variable $X$ having pdf $f(x)$, the $q$-entropy is defined by

$$
I_{q}(\gamma)=\frac{1}{q-1}\left[1-\int_{0}^{\infty} f^{q}(x) d x\right]
$$

where $q>0, q \neq 1$ and $q$ is a real non-integer. 
M.H. Tahir, M. Mansoor, M. Zubair and G.G. Hamedani

Now, taking $q$ th power of McLL density given in (27) as

$$
f_{\gamma}^{q}(x)=\left\{\frac{c}{B\left(a c^{-1}, b\right)}\left(\frac{\alpha}{\beta}\right)\left(\frac{x}{\beta}\right)^{a \alpha-1}\left[1+\left(\frac{x}{\beta}\right)^{\alpha}\right]^{-(a+1)}\left[1-\left\{1-\left[1+\left(\frac{x}{\beta}\right)^{\alpha}\right]^{-1}\right\}^{c}\right]^{b-1}\right\}^{q}
$$

Using (5.3) in (5.6), the expression of $q$-entropy for McLL distribution will be

$$
I_{q}(\gamma)=\frac{1}{q-1}\left[1-\left\{\sum_{j, k=0}^{\infty} V_{j} V_{k}\left(\frac{\alpha}{\beta}\right)^{q-1} B\left(\frac{q(\alpha-1)+1}{\alpha}, q(a+1)+k-\frac{q(\alpha-1)+1}{\alpha}\right)\right\}\right] .
$$

\section{Maximum likelihood estimation}

Here, we consider estimation of the model parameters for $\operatorname{McLL}(a, b, c, \alpha, \beta)$ distribution by the method of maximum likelihood. We assume that $X \sim \operatorname{McLL}(a, b, c, \alpha, \beta)$ and let $\Theta=(a, b, c, \alpha, \beta)$ be the parameter vector of interest. The log-likelihood function $\ell=\ell(\Theta)$ for a random sample $x_{1}, x_{2}, \ldots, x_{n}$ is given by

$$
\begin{aligned}
\ell= & n \log \left[c \alpha \beta^{-1}\left[B\left(\frac{a}{c}, b\right)\right]^{-1}\right]+(a \alpha-1) \sum_{i=1}^{n} \log \left(\frac{x_{i}}{\beta}\right)-(a+1) \sum_{i=1}^{n} \log \left[1+\left(\frac{x_{i}}{\beta}\right)^{\alpha}\right] \\
& +(b-1) \sum_{i=1}^{n} \log \left\{1-\left[1-\left\{1+\left(\frac{x_{i}}{\beta}\right)^{\alpha}\right\}^{-1}\right]^{c}\right\} .
\end{aligned}
$$

Taking partial derivatives of $\ell$ with respect to $a, b, c, \alpha$ and $\beta$, we obtain five equations as

$$
\begin{aligned}
& \frac{\partial \ell}{\partial a}=\frac{-n}{c} \psi\left(a c^{-1}\right)+\frac{n}{c} \psi\left(a c^{-1}+b\right)+\alpha \sum_{i=1}^{n} \log \left(\frac{x_{i}}{\beta}\right)-\sum_{i=1}^{n} \log \left[1+\left(\frac{x_{i}}{\beta}\right)^{\alpha}\right] \\
& \frac{\partial \ell}{\partial b}=-n \psi(b)+n \psi\left(a c^{-1}+b\right)+\sum_{i=1}^{n} \log \left\{1-\left[1-\left\{1+\left(\frac{x_{i}}{\beta}\right)^{\alpha}\right\}^{-1}\right]^{c}\right\} \\
& \frac{\partial \ell}{\partial c}=\frac{n}{c}-\frac{n a}{c^{2}} \psi\left(a c^{-1}\right)+\frac{n a}{c^{2}} \psi\left(a c^{-1}+b\right) \\
& -(b-1) \sum_{i=1}^{n}\left\{\frac{\left[1-\left\{1+\left(\frac{x_{i}}{\beta}\right)^{\alpha}\right\}^{-1}\right]^{c} \log \left[1-\left\{1+\left(\frac{x_{i}}{\beta}\right)^{\alpha}\right\}^{-1}\right]}{1-\left[1-\left\{1+\left(\frac{x_{i}}{\beta}\right)^{\alpha}\right\}^{-1}\right]^{c}}\right\} \\
& \frac{\partial \ell}{\partial \alpha}=\frac{n}{\alpha}+\sum_{i=1}^{n} \log \left(\frac{x_{i}}{\beta}\right)-(a+1) \sum_{i=1}^{n}\left[\frac{\left(\frac{x_{i}}{\beta}\right)^{\alpha} \log \left(\frac{x_{i}}{\beta}\right)}{1+\left(\frac{x_{i}}{\beta}\right)^{\alpha}}\right] \\
& -c(b-1) \sum_{i=1}^{n}\left[\frac{\left[1-\left\{1+\left(\frac{x_{i}}{\beta}\right)^{\alpha}\right\}^{-1}\right]^{c-1}\left\{1+\left(\frac{x_{i}}{\beta}\right)^{\alpha}\right\}^{-2}\left(\frac{x_{i}}{\beta}\right)^{\alpha} \log \left(\frac{x_{i}}{\beta}\right)}{1-\left[1-\left\{1+\left(\frac{x_{i}}{\beta}\right)^{\alpha}\right\}^{-1}\right]^{c}}\right]
\end{aligned}
$$




$$
\begin{aligned}
\frac{\partial \ell}{\partial \beta}= & \frac{n}{\beta}-\frac{n}{\beta}(a \alpha-1)+(a+1) \sum_{i=1}^{n}\left[\frac{\left(\frac{\alpha}{\beta}\right)\left(\frac{x_{i}}{\beta}\right)^{\alpha}}{1+\left(\frac{x_{i}}{\beta}\right)^{\alpha}}\right] \\
& +(b-1) \sum_{i=1}^{n}\left[\frac{\left.c\left[1-\left\{1+\left(\frac{x_{i}}{\beta}\right)^{\alpha}\right\}^{-1}\right]^{c-1}\left\{1+\left(\frac{x_{i}}{\beta}\right)^{\alpha}\right\}^{-2}\left(\frac{\alpha}{\beta}\right)\left(\frac{x_{i}}{\beta}\right)^{\alpha}\right]}{1-\left[1-\left\{1+\left(\frac{x_{i}}{\beta}\right)^{\alpha}\right\}^{-1}\right]^{c}}\right]
\end{aligned}
$$

where $\psi()=.\Gamma(.)^{\prime} / \Gamma($.$) is the digamma function.$

By equating the above equations to zero, we can get MLEs of $a, b, c, \alpha$ and $\beta$ by solving them simultaneously using iterative methods, such as Newton-Raphson, BFGS, SANN, BHHH, NM and L-BFGS-B.

\section{A useful characterization}

In practice, an investigator will be usually interested to know if the model under study fits the requirements of the proposed distribution. To this end, the investigator relies on the characterizations of the distribution which provide conditions under which the underlying distribution is indeed the proposed distribution.

Our characterization will employ an interesting result due to Glänzel (1987) (Theorem 1 below). The advantage of this characterization is that it does not require the explicit closed form of the cumulative distribution function. It also connects the probability density function with a solution of a first order differential equation.

Theorem 1. Let $(\Omega, \mathfrak{F}, \mathbf{P})$ be a given probability space and let $H=[a, b]$ be an interval for some $a<b(a=-\infty, b=\infty$ might as well be allowed). Let $X: \Omega \rightarrow H$ be a continuous random variable with distribution function $F$ and let $q$ and $h$ be two real functions defined on $H$ such that

$$
E[q(X) \mid X \geq x]=E[h(X) \mid X \geq x] \eta(x), \quad x \in H,
$$

is defined for some real function $\eta$. Assume that $q, h \in C^{1}(H), \eta \in C^{2}(H)$ and $F$ is twice continuously differentiable and strictly monotone function on the set $H$. Further, assume that the equation $h \eta=q$ has no real solution in the interior of $H$. Then, $F$ is uniquely determined by the functions $q$, $h$ and $\eta$, particularly

$$
F(x)=C \int_{a}^{x}\left|\frac{\eta^{\prime}(u)}{\eta(u) h(u)-q(u)}\right| \exp [-s(u)] d u,
$$

where $s=s(x)$ is a solution of the differential equation $s^{\prime}(x)=\left[\eta^{\prime}(x) h(x)\right] /[\eta(x) h(x)-q(x)]$ and $C$ is a constant chosen such that $\int_{H} d F=1$.

We mention that this kind of characterization based on the ratio of truncated moments is stable in the sense of weak convergence, in particular, let us assume that there is a sequence $\left\{X_{n}\right\}$ of random variables with distribution functions $\left\{F_{n}\right\}$ such that the functions $q_{n}, h_{n}$ and $\eta_{n}(n \in \mathbb{N})$ satisfy the conditions of Theorem 1 and let $q_{n} \rightarrow q, h_{n} \rightarrow h$ for some continuously differentiable real functions $q$ and $h$. Let $X$ be a random variable with distribution $F$. Under the condition that $q_{n}(X)$ and $h_{n}(X)$ are uniformly integrable and that the family is relatively compact, the sequence $X_{n}$ converges to $X$ 
in distribution if and only if $\eta_{n}$ converges weakly to $\eta$, where

$$
\eta(x)=\frac{E[q(X) \mid X \geq x]}{E[h(X) \mid X \geq x]}
$$

This stability theorem makes sure that the convergence of distribution functions is reflected by corresponding convergence of the functions $q, h$ and $\eta$, respectively. It guarantees, for instance, the "convergence" of characterization of the Wald distribution to that of the Lévy-Smirnov distribution if $\alpha \rightarrow \infty$.

A further consequence of the stability property of Theorem 1 is the application of this theorem to special tasks in statistical practice such as the estimation of the parameters of discrete distributions. For such purpose, the functions $q, h$ and, specially, $\eta$ should be as simple as possible. Since the function triplet is not uniquely determined it is often possible to choose $\eta$ as a linear function. Therefore, it is worth analyzing some special cases which helps to find new characterizations reflecting the relationship between individual continuous univariate distributions in other areas of statistics. In Theorem 1, the interval $H$ need not be closed. The goal is to have the function $\eta$ as simple as possible. For a more detailed discussion on the choice of $\eta$, we refer the reader to Glänzel and Hamedani (2001), and Hamedani (2006).

Proposition 1. Let $X: \Omega \rightarrow(0, \infty)$ be a continuous random variable and let

$h(x)=\left(\frac{x}{\beta}\right)^{\alpha(1-a)}\left[1-\left\{1-\left[1+(x / \beta)^{\alpha}\right]^{-1}\right\}^{c}\right]^{1-b}$ and

$q(x)=\left(\frac{x}{\beta}\right)^{\alpha(2-a)}\left[1-\left\{1-\left[1+(x / \beta)^{\alpha}\right]^{-1}\right\}^{c}\right]^{1-b}$ for $x \in(0, \infty)$.

The pdf of $X$ for $a>1$ is (2.5) if and only if the function $\eta$ defined in Theorem 1 has the form

$$
\eta(x)=\frac{1}{(a-1)}\left[1+a\left(\frac{x}{\beta}\right)^{\alpha}\right], \quad x>0 .
$$

Proof. Let $X$ has pdf (2.5), then

$$
[1-F(x)] \mathbf{E}[h(X) \mid X \geq x]=\frac{c}{a B\left(a c^{-1}, b\right)}\left[1+\left(\frac{x}{\beta}\right)^{\alpha}\right]^{-a}, \quad x>0,
$$

and

$$
[1-F(x)] \mathbf{E}[q(X) \mid X \geq x]=\frac{c}{a(a-1) B\left(a c^{-1}, b\right)}\left[1+\left(\frac{x}{\beta}\right)^{\alpha}\right]^{-a}\left[1+a\left(\frac{x}{\beta}\right)^{\alpha}\right], \quad x>0 .
$$

Finally,

$$
\eta(x) h(x)-q(x)=\frac{h(x)}{a-1}\left[1+\left(\frac{x}{\beta}\right)^{\alpha}\right]>0, \quad a>1, x>0 .
$$

Conversely, if $\eta$ is given as above, then

$$
s^{\prime}(x)=a\left(\frac{\alpha}{\beta}\right)\left(\frac{x}{\beta}\right)^{\alpha-1}\left[1+\left(\frac{x}{\beta}\right)^{\alpha}\right]^{-1},
$$

and hence 


$$
s(x)=\log \left\{\left[1+\left(\frac{x}{s}\right)^{\alpha}\right]^{a}\right\}, x>0 .
$$

Now, in view of Theorem 1, $X$ has pdf (2.5).

Remark 1. Clearly, there are other triplets $(h, q, \eta)$ satisfying the conditions of Proposition 1.

Corollary 1. Let $X: \Omega \rightarrow(0, \infty)$ be a continuous random variable and let $h(x)$ be as in Proposition 1. The pdf of $X$ for $a>1$ is (1.9) if and only if there exist functions $q$ and $\eta$ defined in Theorem 1 satisfying the differential equation

$$
\frac{\eta^{\prime}(x) h(x)}{\eta(x) h(x)-q(x)}=\frac{\alpha a x^{\alpha-1}}{\beta^{\alpha}}\left[1+\left(\frac{x}{\beta}\right)^{\alpha}\right]^{-1}, \quad x>0 .
$$

Remark 2. The general solution of the differential equation given in Corollary 1 is

$\eta(x)=\left[1+\left(\frac{x}{\beta}\right)^{\alpha}\right]^{a}\left[-\frac{\alpha a}{\beta} \int q(x)\left(\frac{x}{\beta}\right)^{\alpha a-1}\left[1+\left(\frac{x}{\beta}\right)^{\alpha}\right]^{-(a+1)}\left[1-\left\{1-\left[1+\left(\frac{x}{\beta}\right)^{\alpha}\right]^{-1}\right\}^{c}\right]^{b-1} d x+D\right]$, for $x>0$, where $D$ is a constant. To see this note that (7.1) can be written as

$$
\eta^{\prime}(x)-\eta(x) \frac{\alpha a x^{\alpha-1}}{\beta^{\alpha}}\left[1+\left(\frac{x}{\beta}\right)^{\alpha}\right]^{-1}=-q(x)(h(x))^{-1} \frac{\alpha a x^{\alpha-1}}{\beta^{\alpha}}\left[1+\left(\frac{x}{\beta}\right)^{\alpha}\right]^{-1} .
$$

Now, replacing $h(x)$ from Proposition 1 and then multiplying both sides of (7.2) by $\left[1+\left(\frac{x}{\beta}\right)^{\alpha}\right]^{-a}$, we obtain

$$
\frac{d}{d x}\left\{\eta(x)\left[1+\left(\frac{x}{\beta}\right)^{\alpha}\right]^{-a}\right\}=-\frac{\alpha a}{\beta} q(x)\left(\frac{x}{\beta}\right)^{\alpha a-1}\left[1+\left(\frac{x}{\beta}\right)^{\alpha}\right]^{-(a+1)}\left[1-\left\{1-\left[1+\left(\frac{x}{\beta}\right)^{\alpha}\right]^{-1}\right\}^{c}\right]^{b-1},
$$

from which the expression for $\eta(x)$ given above will follow. One set of appropriate functions is given in Proposition 1 with $D=0$.

\section{An application}

In this section, we present an application of McLL to breast cancer data to illustrate its potentiality. The MLEs of real data set are computed, and goodness-of-fit of McLL is compared with the other competing models.

The real data set represent the survival times of 121 patients with breast cancer obtained from a large hospital in a period from 1929 to 1938 (Lee, 1992). This data set has recently been studied by Ramos et al. (2013). The data are: 0.3, 0.3, 4.0, 5.0, 5.6, 6.2, 6.3, 6.6, 6.8, 7.4, 7.5, 8.4, 8.4, 10.3, $11.0,11.8,12.2,12.3,13.5,14.4,14.4,14.8,15.5,15.7,16.2,16.3,16.5,16.8,17.2,17.3,17.5$, 17.9, 19.8, 20.4, 20.9, 21.0, 21.0, 21.1, 23.0, 23.4, 23.6, 24.0, 24.0, 27.9, 28.2, 29.1, 30.0, 31.0, $31.0,32.0,35.0,35.0,37.0,37.0,37.0,38.0,38.0,38.0,39.0,39.0,40.0,40.0,40.0,41.0,41.0$, 41.0, 42.0, 43.0, 43.0, 43.0, 44.0, 45.0, 45.0, 46.0, 46.0, 47.0, 48.0, 49.0, 51.0, 51.0, 51.0, 52.0, 54.0, 55.0, 56.0, 57.0, 58.0, 59.0, 60.0, 60.0, 60.0, 61.0, 62.0, 65.0, 65.0, 67.0, 67.0, 68.0, 69.0, 78.0, 80.0,83.0, 88.0, 89.0, 90.0, 93.0, 96.0, 103.0, 105.0, 109.0, 109.0, 111.0, 115.0, 117.0, 125.0, 126.0, 127.0, 129.0, 129.0, 139.0, 154.0.

In the following, we compare McLL distribution with other seven lifetime models Viz. McDonald Weibull (McW), ZBLL, BLL, KwLL, LL, Gamma (Ga) and Log-Normal (LN). The pdf of McW is given Cordeiro et al. (2012). 
We estimate the unknown parameters of each model by the maximum likelihood. There exists many maximization methods in R Packages like NR (Newton-Raphson), BFGS (Broyden-FletcherGoldfarb-Shanno), BHHH (Berndt-Hall-Hall-Hausman), SANN (Simulated-Annealing) and NM (Nelder-Mead). But here the maximum likelihood estimates (MLEs) are computed using LimitedMemory quasi-Newton code for Bound-constrained optimization (L-BFGS-B) and the measures of goodness of fit AIC, CAIC, BIC, Anderson-Darling $\left(A^{*}\right)$ and Cramér-von Mises $\left(W^{*}\right)$ are used to compare the eight models. The statistics $W^{*}$ and $A^{*}$ are described in details in Chen and Balakrishnan (1995). In general, the smaller the values of goodness-of-fit measure suggest the better the fit to the data. The required computations are carried out using R-package AdequacyModel which is recently introduced by Pedro Rafael Diniz Marinho and Cícero Rafael Barros Dias and is freely available from

http://cran.r-project.org/web/packages/AdequacyModel/AdequacyModel.pdf.

Table 1. MLEs and their standard errors (in parentheses) for Breast Cancer Data

\begin{tabular}{lccccccc}
\hline Distribution & $a$ & $b$ & $c$ & $\alpha$ & $\beta$ & $\mu$ & $\sigma$ \\
\hline $\operatorname{McW}(a, b, c, \alpha, \beta)$ & 0.961 & 2.926 & 38.441 & 1.250 & 0.018 & - & - \\
& $(0.483)$ & $(3.953)$ & $(36.188)$ & $(0.429)$ & $(0.0 .006)$ & - & - \\
$\operatorname{ZBLL}(a, \alpha, \beta)$ & 0.353 & 77.856 & 3.098 & - & - & - & - \\
$\operatorname{BLL}(a, b, \alpha, \beta)$ & $(0.103)$ & $(12.562)$ & $(0.579)$ & - & - & - & - \\
& 0.364 & 0.732 & 53.251 & 3.368 & - & - & - \\
$\operatorname{McLL}(a, b, c, \alpha, \beta)$ & $(0.230)$ & $(0.482)$ & $(9.731)$ & $(1.176)$ & - & - & - \\
$\operatorname{LL}(\alpha, \beta)$ & $(0.216)$ & $(78.676$ & 30.988 & 2.272 & 65.025 & - & - \\
& - & - & - & 1.856 & 35.177 & - & - \\
$\operatorname{KwLL}(a, b, \alpha, \beta)$ & - & - & - & $(0.141)$ & $(2.978)$ & - & - \\
$\operatorname{Ga}(\alpha, \beta)$ & $(4.804)$ & $(13.979)$ & $(0.336$ & 0.044 & - & - & - \\
$\operatorname{LN}(\mu, \sigma)$ & - & - & - & 1.495 & 30.984 & - & - \\
& - & - & - & $(0.175)$ & $(4.290)$ & - & - \\
& - & - & - & - & - & 3.46 & 1.033 \\
& - & - & - & - & - & $(0.094)$ & $(0.066)$ \\
\hline
\end{tabular}

Table 1 lists the MLEs and their corresponding standard errors (in parentheses) of the model parameters. The model selection is carried out using the following statistics: AIC (Akaike information criterion), CAIC (consistent Akaike information criterion) and BIC (Bayesian information criterion): $A I C=-2 \ell()+.2 p, C A I C=-2 \ell()+.\frac{2 p n}{n-p-1}$ and $B I C=-2 \ell()+.p \log n$, where $\ell($. denotes the log-likelihood function evaluated at the maximum likelihood estimates, $p$ is the number of parameters, and $n$ is the sample size. The numerical values of statistics AIC, CAIC, BIC, $W^{*}$ and $A^{*}$ are listed in Table 2 .

We noted from Table 2 that McLL model shows lowest values of AIC and CAIC among the fitted models: McW, ZBLL, BLL, LL, KwLL, Ga and LN, suggesting that the McLL distribution shows the best fit, and therefore could be chosen as the best model. Further, the value of $A^{*}$-statistic for McLL model is also smaller as compared to the other models especially to the ZBLL, suggesting that McLL distribution seems to be a competitive model for the cancer data. The histogram of McLL 
estimated pdf and plot of McLL estimated cdf are displayed in Figure 3. It is clear from Table 2 and Figure 3 that McLL distribution provided a better fit and therefore be one of the best models for cancer data.

Table 2. The AIC, CAIC, BIC, $A^{*}$ and $W^{*}$ values for Breast Cancer data

\begin{tabular}{lccccc}
\hline \multirow{2}{*}{ Distribution } & AIC & CAIC & BIC & $A^{*}$ & $W^{*}$ \\
\hline \multirow{2}{*}{ McLL } & 1164.661 & 1165.183 & 1178.640 & 0.401 & 0.058 \\
McW & 1166.474 & 1166.996 & 1180.453 & 0.513 & 0.074 \\
ZBLL & 1167.063 & 1167.268 & 1175.450 & 0.454 & 0.053 \\
BLL & 1171.861 & 1172.206 & 1183.045 & 0.494 & 0.066 \\
LL & 1179.199 & 1184.791 & 1179.301 & 1.258 & 0.209 \\
KwLL & 1189.937 & 1190.282 & 1201.120 & 1.511 & 0.232 \\
Ga & 1166.474 & 1166.996 & 1180.453 & 0.513 & 0.074 \\
LN & 1194.067 & 1194.168 & 1199.658 & 2.043 & 0.318 \\
\hline
\end{tabular}

(a) Estimated pdfs

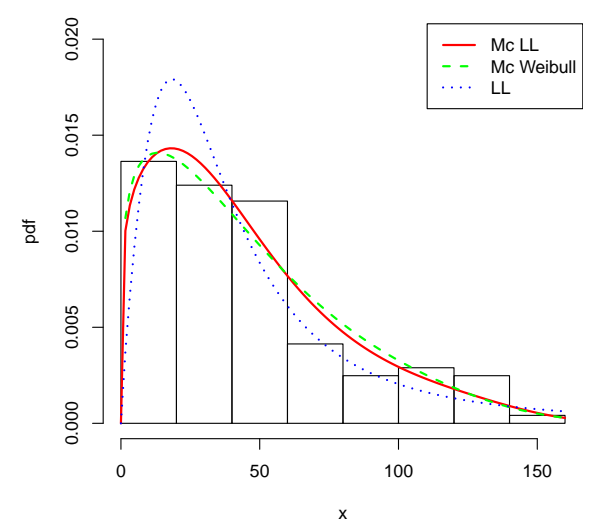

(b) Estimated cdfs

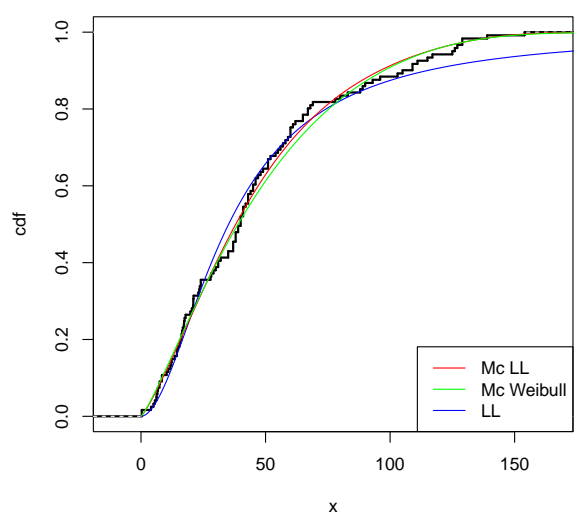

Fig. 3. Plots of the estimated pdfs and cdfs McLL, McW and LL models

\section{Concluding remarks}

In this paper, we propose a new five parameter McLL distribution which generalizes LL distribution, and we also obtained some of its structural properties. We provide for the new distribution: the expression for mean residual life, mean waiting time, Rényi entropy and $q$-entropy. The model parameters are estimated by the maximum likelihood. The usefulness of the new model is illustrated in an application to real data set on breast cancer by using five goodness of fit measures. The new model provides consistently better fit than the other seven models selected from the literature. We hope that the proposed model may attract wider application in many areas such as engineering, survival data, hydrology, economics (income inequality), and others.

Addendum. After the completion of the present work we came across a paper by Cordeiro et al. (to appear in 2014) in which the authors have employed $G(x)=\left\{1-\left[1+\left(\frac{x}{\beta}\right)^{\alpha}\right]^{-k}\right\}$. The properties discussed in that paper, however, are different from ours. 


\section{Acknowledgments}

The authors wish to thank the Editor-in-Chief, the Editor and the two anonymous referees for their comments and suggestions which have greatly improved our manuscript.

\section{References}

[1] Alexander, C., Cordeiro, G.M., Ortega, E.M.M. and Sarabia, J.M. (2012). Generalized beta-generated distributions. Computational Statistics and Data Analysis, 56, 1880-1897.

[2] Chen, G. and Balakrishnan, N. (1995). A general purpose approximate goodness-of-fit test. Journal of Quality Technology, 27, 154-161

[3] Corderio, G.M. and de Castro, M. (2011). A new family of generalized distributions. Journal of Statistical Computation and Simulation, 81, 883-893.

[4] Corderio, G.M., Hashimoto, E.H. and Ortega, E.M.M. (2012). The McDonald Weibull model. Statistics. iFirst. DOI:10.1080/02331888.2012.748769

[5] Corderio, G.M., Ortega, E.M.M., Hamedani, G.G. and Gracia, D.A. (2014). The McDonald Burr XII model: Properties and Applications. (To appear)

[6] Eugene, N., Lee, C. and Famoye, F. (2002). Beta-normal distribution and its applications. Communications in Statistics-Theory and Methods, 31, 497-512.

[7] Fisk, P.R. (1961). The graduation of income distribution. Econometrica, 29, 171-185.

[8] Glänzel, W. (1987). A characterization theorem based on truncated moments and its application to some distribution families. In Mathematical Statistics and Probability Theory (Bad Tatzmannsdorf, 1986), Vol. B, Reidel, Dordrecht, pp. 75-84.

[9] Glänzel, W. and Hamedani, G.G. (2001). Characterizations of univariate continuous distributions. Studia Scientiarum Mathematicarum Hungarica, 37, 83-118.

[10] Hamedani, G.G. (2006). Characterizations of univariate continuous distributions-III. Studia Scientiarum Mathematicarum Hungarica, 43, 361-385.

[11] Havrda, J. and Charvat, F. (1967). Quantification method in classification processes: concept of structural $\alpha$-entropy. Kybernetika, 3, 30-35.

[12] Jones, M.C. (2004). Families of distributions arising from the distributions of order statistics. Test, 13, $1-43$.

[13] Lee, E.T. (1992) Statistical Methods for Survival Data Analysis. John Wiley: New York.

[14] Lemonte, A.L. (2012). The beta log-logistic distribution. To appear in Brazilian Journal of Probability and Statistics-BJPS-209.

[15] McDonald, J.B. (1984). Some generalized functions for the size distribution of income. Econometrica, 52, 647-663.

[16] Ramos, M.W.A., Cordeiro, G.M., Marinho, P.R.D., Dias, C.R.B. and Hamedani, G.G. (2013). The Zografos-Balakrishnan log-logistic distribution: Properties and applications. Journal of Statistical Theory and Applications, 12, 225-244.

[17] Rényi, A. (1961). On measures of entropy and information. University of California Press, Berkeley, California, pp. 547-561.

[18] de Santana, T.V.F., Ortega, E.M.M., Cordeiro, G.M. and Silva, G.O. (2012). The Kumaraswamy-logLogistic distribution. Journal of Statistical Theory and Applications, 11, 265-291.

[19] Tsallis, C. (1988). Possible generalization of Boltzmann-Gibbs statistics. Journal of Statistical Physics, 52, 479-487.

[20] Ullah, A. (1996). Entropy, divergence and distance measures with econometric applications. Journal of Statistical Planning and Inference, 49, 137-162.

[21] Verhulst, P.F. (1838). Notice sur la loi que la population suit dans son accroissement. Correspondence math $m$ atrique et physique, 10, 113-121.

[22] Zografos, K. and Balakrishnan, N. (2009). On families of beta- and generalized gamma-generated distributions and associated inference. Statistical Methodology, 6, 344-362. 